LAWRENCE LIVERMORE NATIO NAL LABORATORY

\title{
Progress in Inertial Fusion Energy Technology: June - September 2003
}

W. R. Meier, J. F. Latkowski, R. P. Abbott, S. Reyes, R. W. Moir, J. Yuan, G. A. Moses, Z. Gui, G. L. Kulcinski, P. F. Peterson, J. Freeman, P. Bardet, C. Debonnel, G. Fukuda, H. Zhao, D. Olander, M. Abdou, A. Ying, N. Morley, P. Calderoni, A. Konkachbaev, A. Elias, M. Ni, T. Sketchley, S. G. Durbin, M. Yoda, S. I. Abdel-Khalik, D. Goodin, A. Nobile, R. Petzoldt, B. Vermillion, G. Besenbruch, J. Maxwell, M. S. Tillack, F. Najmabadi, A. R. Raffray, S. S. Harilal, C. V. Bindhu, A. C. Gaeris, M. R. Zaghloul, J. O'Shay, K. Sequoia

October 27, 2003 
This document was prepared as an account of work sponsored by an agency of the United States Government. Neither the United States Government nor the University of California nor any of their employees, makes any warranty, express or implied, or assumes any legal liability or responsibility for the accuracy, completeness, or usefulness of any information, apparatus, product, or process disclosed, or represents that its use would not infringe privately owned rights. Reference herein to any specific commercial product, process, or service by trade name, trademark, manufacturer, or otherwise, does not necessarily constitute or imply its endorsement, recommendation, or favoring by the United States Government or the University of California. The views and opinions of authors expressed herein do not necessarily state or reflect those of the United States Government or the University of California, and shall not be used for advertising or product endorsement purposes.

This work was performed under the auspices of the U.S. Department of Energy by University of California, Lawrence Livermore National Laboratory under Contract W-7405-Eng-48. 


\title{
Progress in Inertial Fusion Energy Technology: June - September 2003
}

\author{
Compiled by S. Reyes
}

Please direct comments and/or contributions to

Work of LLNL contributors performed under the auspices of the U.S. Department of Energy by University of California, Lawrence Livermore National Laboratory under contract No. W-7405-Eng-48. 


\section{Progress in IFE Technology: June-September 2003}

\section{Integration, Systems Studies, Safety \& Environment and Driver-Chamber Interface - Lawrence Livermore National Laboratory}

W. R. Meier, J. F. Latkowski, R. P. Abbott, S. Reyes and R. W. Moir

- Completed IFSA paper on fast ignition. Found that for a HIF plant, driver can be reduced from 7 MJ to 2.75 MJ and from 120 beams to 48 beams. The COE is reduced by about $25 \%$ (see Figure 1 ).

- Compiled a thermo-mechanical stress analysis of proposed high-temperature C/C$\mathrm{SiC}$ compact heat exchangers for use in molten salt protected IFE power plants (see Figures 2 and 3).

- Completed multimaterial version of ABLATOR code for modeling of x-ray ablation on IFE first wall and optics, paper presented at IFSA conference.

- Performed safety analysis of beryllium use in IFE, compared radioactivity versus chemical toxicity hazards. Results show that beryllium chemical exposures would be more hazardous that radioactive doses in case of accidental release of activated flibe.

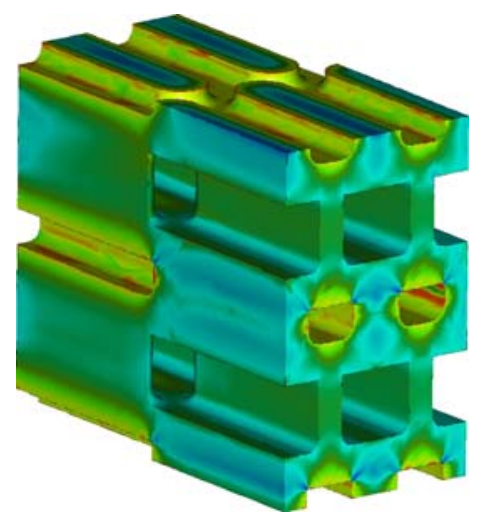

Figure 2. Maximum principal stress distribution in heat exchanger element

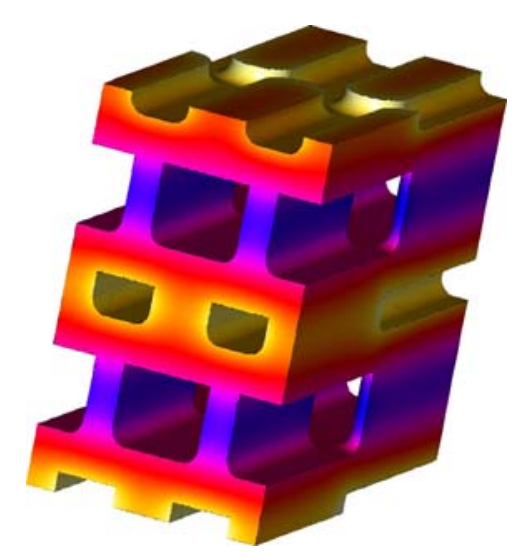

Publications and Presentations

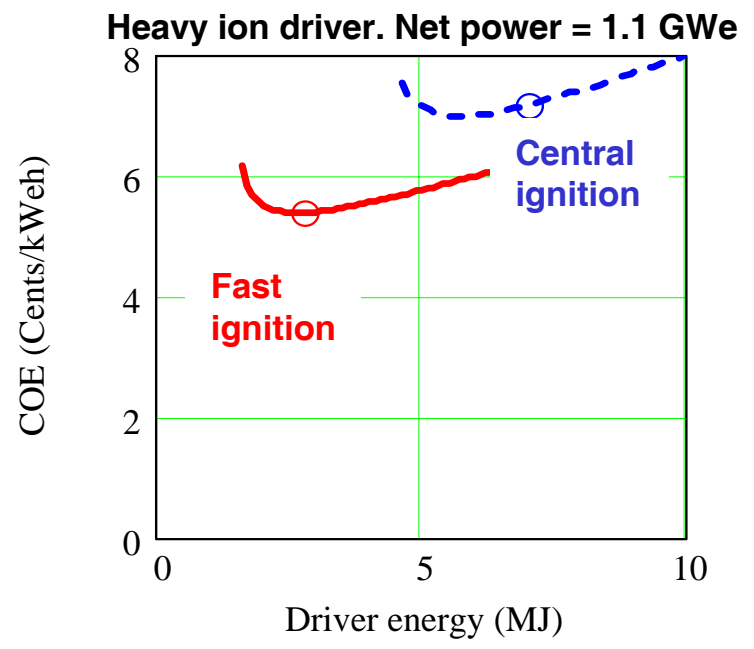

Figure 1. COE for HIF plant, fast ignition versus conventional central ignition

Figure 3. Temperature distribution in heat exchanger element

[1] W.J. Hogan, W.R. Meier , “Technology Issues and Benefits of a Fast Ignition Power Plant with Cone Targets.” Presented at IFSA2003.

[2] S. Reyes, J. F. Latkowski, R. P. Abbott, W. Stein, "Simulation of X-Ray Irradiation on Optics and Chamber Wall Materials for Inertial Fusion Energy.” Presented at IFSA2003.

[3] J. F. Latkowski, R. P. Abbott, S. A. Payne, S. Reyes, R. C. Schmitt, J. A. Speth, "Rep-Rated X-Ray Damage and Ablation Experiments for IFE and ICF Applications.” Presented at IFSA2003.

[4] S. Reyes, L. C. Cadwallader, J. F. Latkowski, J. Gomez Del Rio, J. Sanz, "Safety Issues of Beryllium Use in IFE Power Plants.” Presented at the 20th IEEE/NPSS Symposium on Fusion Engineering (SOFE), October 14 - 17, 2003, San Diego, CA. 


\section{Progress in IFE Technology: June-September 2003 (Cont'd.)}

IFE Liquid Wall Chamber Dynamics / Tools for Atomic Data and opacities - University of Wisconsin - Madison

\section{J. Yuan, G. A. Moses, Z. Gui and G. L. Kulcinski}

- The infrastructure has been built to integrate the graphical user interface (in Java) with the underlying native atomic codes written in Fortran. Java Native Interface and process creation technology are applied for communication between the Java user interfaces and the Fortran codes.

- The NCSA Hierarchical Data Format (HDF5) has been successfully used as the data format standard for storing the various atomic data, including the wave functions, transition energies, oscillator strength and so on.

- A preliminary web site ( http://lapop.ep.wisc.edu:8990/Webatom-root/index.jsp) aimed to provide useful tools for atomic data and spectrum analysis online has been set up. Further work is required for practical use.

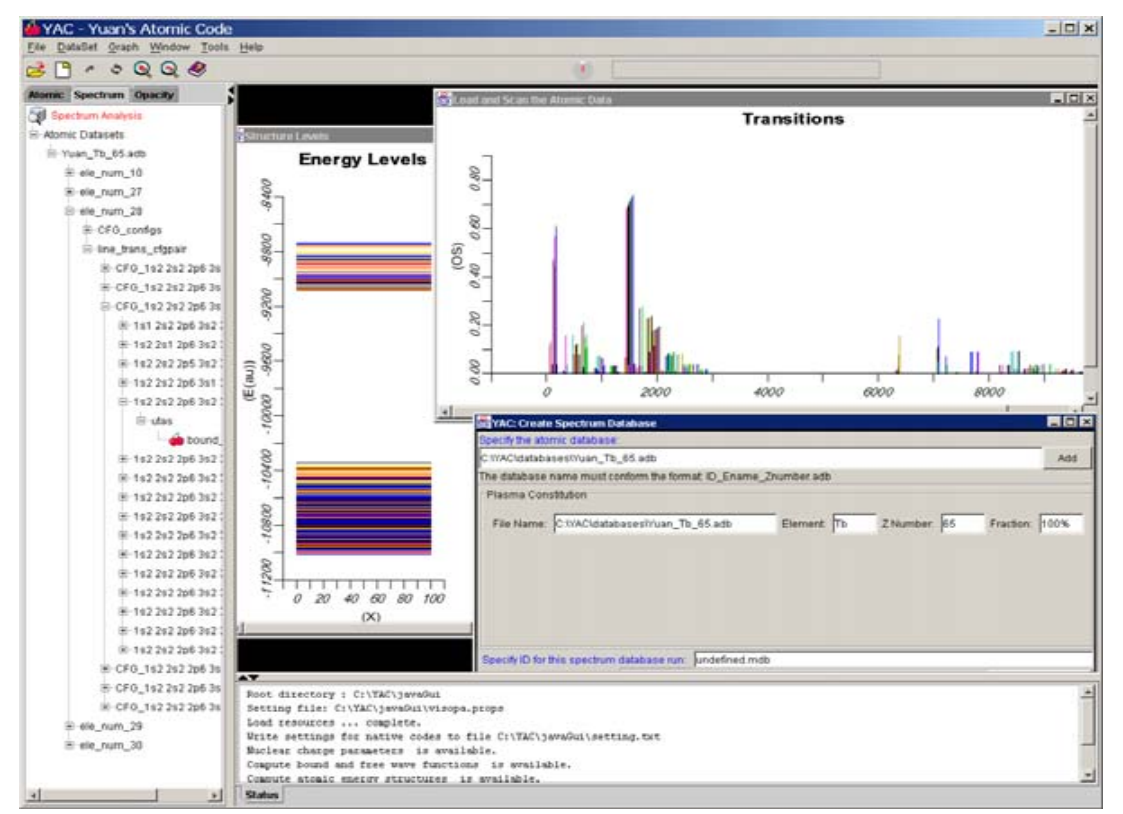

Figure 1. Screenshot of the YAC code to display the energy structures and line transitions.
- Tasks accomplished for developing the YAC code:

1) Capable to calculate the relativistic wave functions for any elements in the periodic table and to plot in graphs.

2) Capable to compute the energy level structures under two different atomic models, the detailed term accounting (DTA) model and the relativistic single-configuration singletransition unresolved transition (RSSUTA) model.

3) Capable to compute the oscillator strengths between the energy levels under the RSSUTA model.

- Future work:

1) Photo-ionization calculations under the self-consistent field approximation.

2) Free-free bremstrahlung calculations.

3) Incorporate these three major components (bound-bound, photo-ionization, bremstrahlung) to calculate the spectrum and opacity.

\section{Publications and Presentations:}

[1] Jiankui Yuan, and Gregory Moses, Atomic Physics of Plasma Database Project, DOE Review, Gaitherburg, MD, July 23, 2003

[2] Jiankui Yuan, Building Tools for Atomic Data Calculations, poster presented at the High Average Power Laser Program Meeting, University of Wisconsin, 24-25 September, 2003. 


\section{Progress in IFE Technology: June-September 2003 (Cont'd.)}

Thick-Liquid Protection-University of California at Berkeley

\section{P. F. Peterson, J. Freeman, P. Bardet, C. Debonnel, G. Fukuda, H. Zhao, D. Olander}
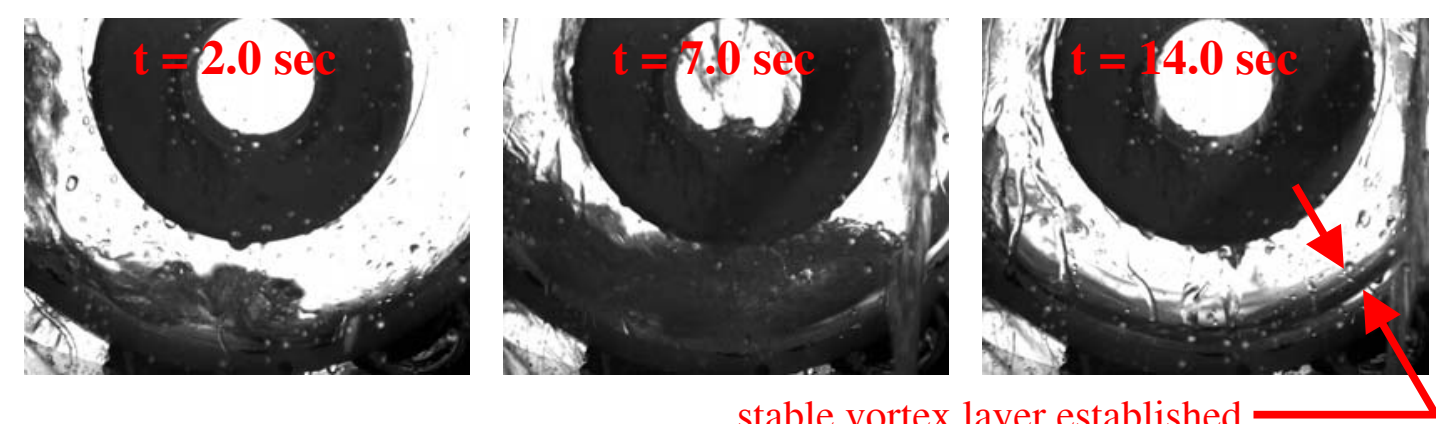

stable vortex layer established

- UC Berkeley has extended its earlier work on HIF beam-tube vortexes to larger-scale vortices by a combination of wall blowing and suction. Initial experiments with a $22-\mathrm{cm}$ diameter device demonstrated that the liquid pool that initially accumulates at the bottom of the vortex chamber can be cleared by a phased startup of injection nozzles, forming a stable vortex layer with a thickness between 1.0 and $3.0 \mathrm{~cm}$ (thickness up to $25 \%$ of the chamber radius) depending on the number of injection nozzles running. In initial experiments, stable operation with Froude numbers $\left(\mathrm{V}^{2} / \mathrm{Rg}\right)$ as low as 4.0 have been obtained. Due to the kinetic energy injected by the blowing jets, turbulence intensity in these vortex flows is expected to be substantially larger than in the fully-developed turbulent film flows that have been studied previously for MFE chamber protection. Upcoming work will use particle image velocimetry to measure turbulence intensity in beam-tube vortex flow, and further work will be performed to refine the control of large vortex flows using combined blowing and suction.

\section{$\underline{\text { Publications and Presentations }}$}

[1] C.S. Debonnel and P.F. Peterson, "Revisited TSUNAMI simulations for the NIF mini-chamber," submitted to the Third International Conference on Inertial Fusion Sciences and Applications (IFSA2003), Monterey, CA, September 7-12, 2003.

[2] C.S. Debonnel, S. Yu and P.F. Peterson, "Evaporation, Venting, and Condensation for the HIF Robust Point Design," submitted to the Third International Conference on Inertial Fusion Sciences and Applications (IFSA2003), Monterey, CA, September 7-12, 2003. 


\section{Progress in IFE Technology: September 2002 - January 2003 (Cont’d.)}

Vapor Dynamics and Condensation and Free Surface Flow Studies - University of California, Los Angeles

M. Abdou, A. Ying, N. Morley, P. Calderoni, A. Konkachbaev, A. Elias, M. Ni, T. Sketchley

\section{Experimental effort:}

We completed the investigation of transient condensation of lithium fluoride vapors in conditions relevant to IFE systems chamber. We achieved clean, high-vacuum conditions before vapor injection. The brittle nature of the ablated LiF crystal does not allow to use it as discharge confining material in the vapor source. High temperature ceramics also proved to be incompatible with the viscous and thermal shock developed in the high current arc thus we had to rely on polycarbonate material. Despite the presence of traces of non-condensable gases generated by the ablation of the poly-carbonate, the results show that LiF vapors are cleared from the chamber volume in less then 20 ms, as can be seen in Figure 1.

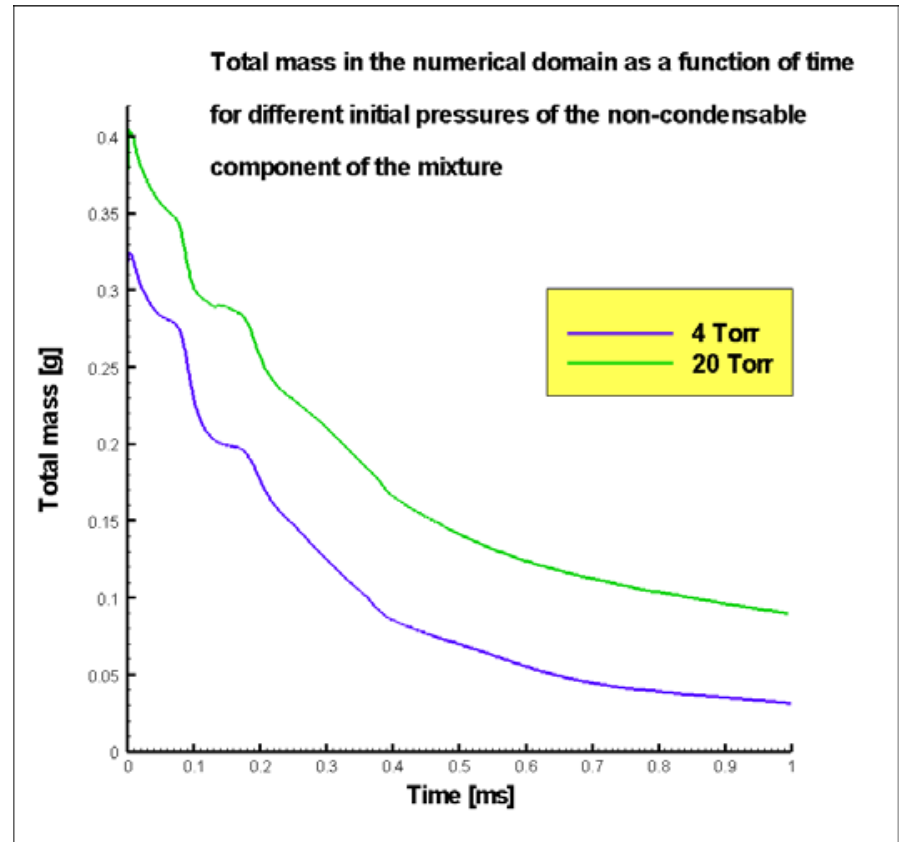

Figure 2. Numerical results of Tsunami simulations with condensation at the boundaries

\section{Modeling:}

\section{Total pressure data for LiF shot of different energy}

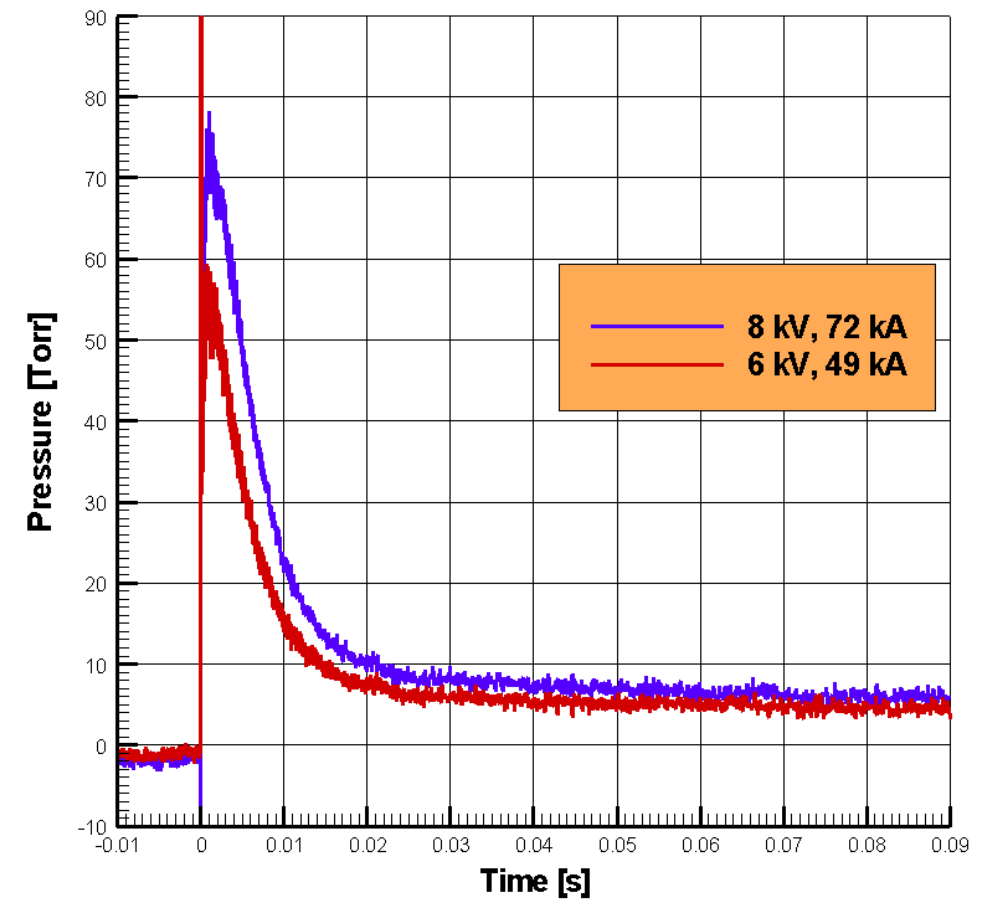

Figure1. Pressure history for different amount of injected material

In order to apply the Tsunami code with condensation at the boundaries to the conditions encountered in the experiments, we modified the condensation subroutine to account for the presence of a mixture of gases, one component of which does not condense. Preliminary results of the decrease of the total mass present in the numerical domain due to condensation in the first millisecond are showed in Figure 2. 


\section{Progress in IFE Technology: June-September 2003 (Cont'd.)}

\section{Thick-Liquid Protection - Georgia Institute of Technology}

S.G. Durbin, M. Yoda, S.I. Abdel-Khalik

- Quantified impact of boundary-layer cutting and flow straightener modifications at $R e=130,000$

- "Hydrodynamic source term" (droplet formation due to turbulent breakup)

- Surface ripple

- BL cutting of well-conditioned flow appears to eliminate hydrodynamic source term

- Boundary layer cutting, see Figure 1:

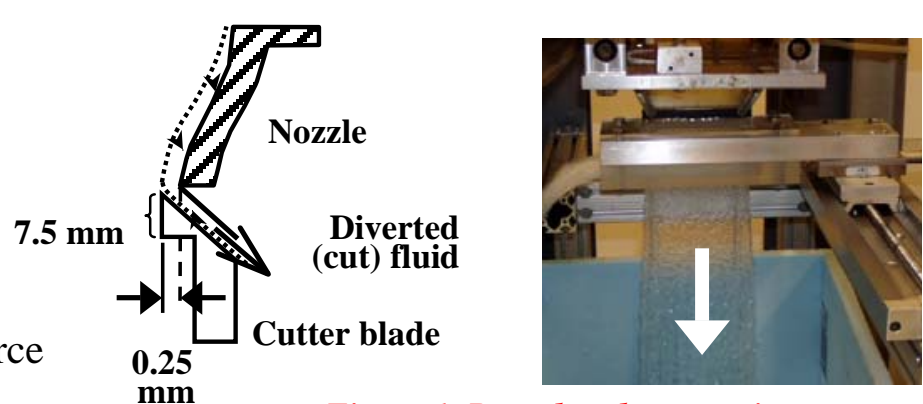

- "Cut" away $0.25 \mathrm{~mm}$ BL fluid on one side of liquid sheet at various $x$-locations

- $\quad$ Removed liquid $(\sim 0.18 \mathrm{~kg} / \mathrm{s})$ diverted to side

- $\quad$ Mass Flux $(\mathrm{x} / \delta=25)$, see Figure 2:

- Hydrodynamic source term sensitive to initial conditions

- Good flow conditioning more important than BL cutting

- Preventing blockage of fine screens major issue

- $\quad$ Surface Ripple $(\mathrm{x} / \delta=25)$, see Figure 3:

- Std. deviation $\sigma_{z}$ averaged over central $75 \%$ of flow

- BL cutting reduces surface ripple by $17 \%$ for standard flow conditioning

- Fine screen has larger impact on flow

\section{$1 \mathbf{c m}$ \\ Figure 4. Cross-section from averaging 130 PLIF images over $4.3 \mathrm{~s}$ \\ - Sharp protrusions near edges of cut jet \\ - Cut jet has smaller y-extent \\ Publications and Presentations
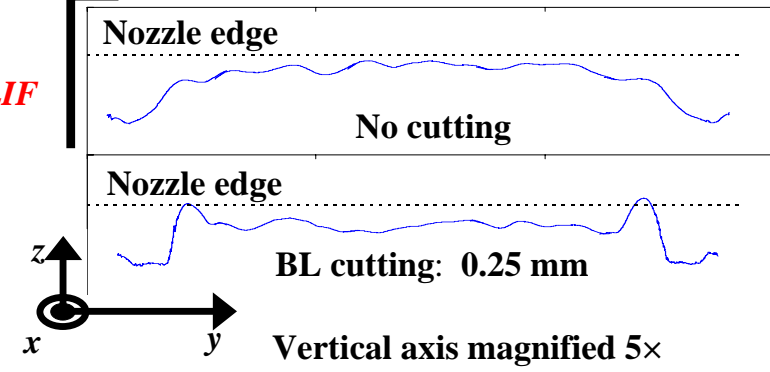

[1] S.G. Durbin, et al., "Surface fluctuation analysis for turbulent liquid sheets," to appear in Fusion Science and Technology (2003)

[2] S. G. Durbin, et al., "The hydrodynamic source term: boundary layer cutting and flow conditioning," ARIES Summer Quarter Project Meeting, Atlanta, GA (2003)

[3] S. I. Abdel-Khalik,et al., "Chamber clearing: the hydrodynamic source term," ARIES Town Meeting on Liquid Wall Chamber Dynamics, Livermore, CA (2003)

[4] S. I. Abdel-Khalik and M. Yoda, "Fluid dynamic aspects of thin liquid film protection concepts," Ibid.
Figure 1. Boundary layer cutting

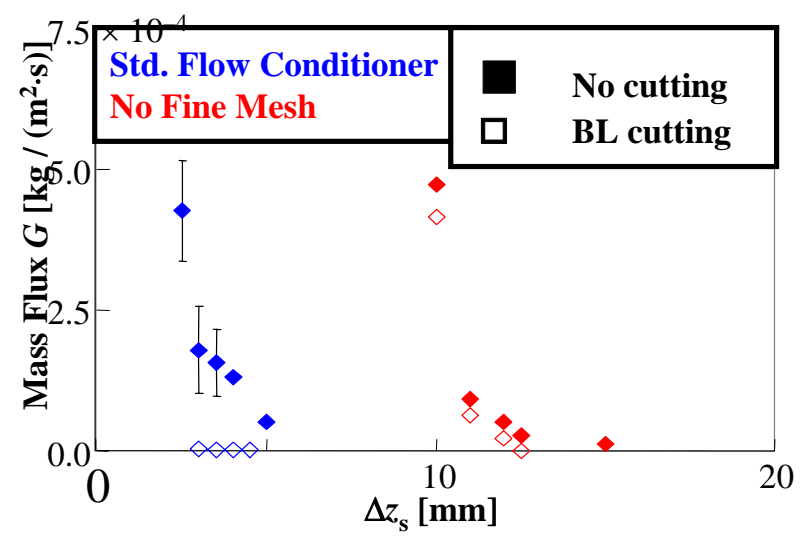

Figure 2. Impact of BL cutting and flow conditioning on hydrodynamic source term

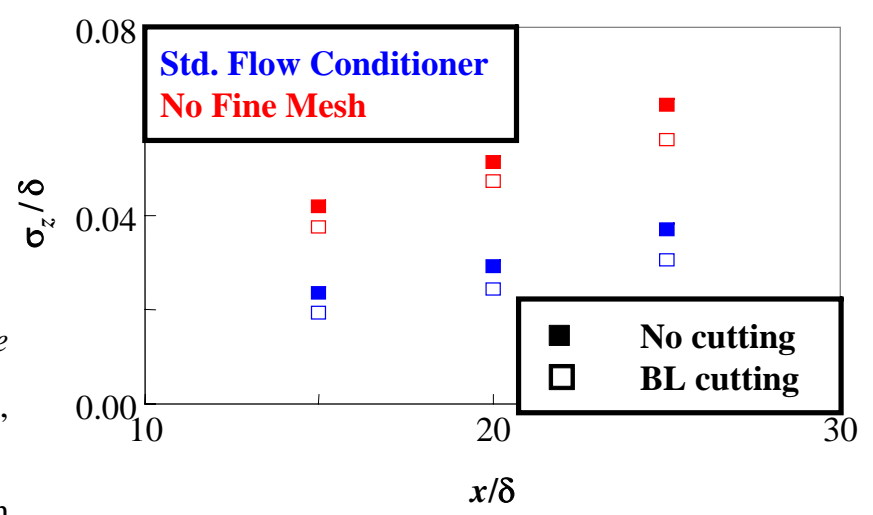

Figure 3. Impact of BL cutting and flow conditioning on surface ripple 


\section{Progress in IFE Technology: June-September 2003 (Cont'd.)}

\section{Target Fabrication, Injection, and Tracking - GA/LANL}

D. Goodin, A. Nobile, R. Petzoldt, B. Vermillion and G. Besenbruch

- Developed a new method to expand the range of possible hohlraum materials in Flibe to include materials that are solid at Flibe operating temperatures. Very fine seed particles ( $\sim .5$ micron) provide a large surface area for plating to occur thereby minimizing plating in small fluid openings (eg. screens and tritium vacuum disengagers) in a liquid wall power plant. The particles would be filtered in a slip stream after growing to about 1.5 micron.

- Completed the design and ordered a unique rifled barrel for hohlraum injection system.

- Evaluated several sabot materials for acceptable operation at up to $400 \mathrm{~m} / \mathrm{s}$ in our $8 \mathrm{~m}$ gun barrel, achieved sabot separation and deflection at over $300 \mathrm{~m} / \mathrm{s}$, and shot targets the full $17 \mathrm{~m}$ length of the system beyond the barrel.

- Calculated radial displacement of membrane supported capsule in rotating hohlraum (see sketch below) during injection. If the capsule is attached to the membrane, additional displacement due to rotation induced stretching of the membrane is negligible.

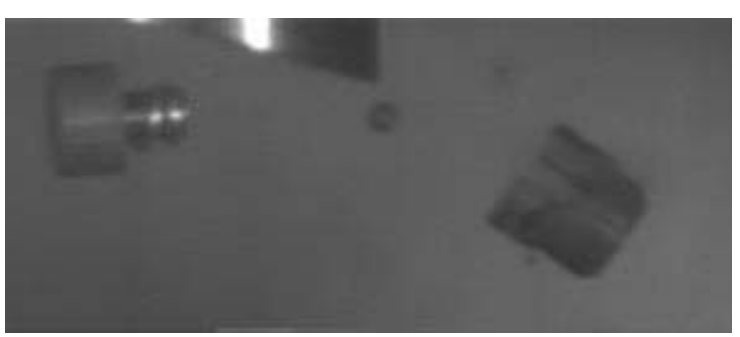

Figure 1. Sabot deflection at over $300 \mathrm{~m} / \mathrm{s}$

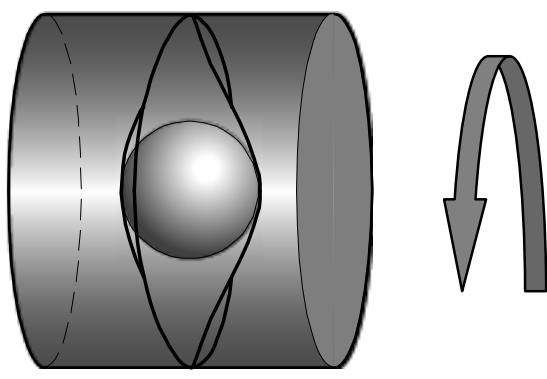

Figure 2. Capsule displacement due to rotation induced membrane stretch is negligible

Publications and Presentations

ARIES:

[1] Ron Petzoldt, "Heavy Ion Fusion Target Materials Selection and Removal of Tungsten Carbide from Flibe.”

IFSA:

[2] Ron Petzoldt, "Experimental Target Injection and Tracking System Construction and Single Shot Testing”

[3] Dan Goodin, “Cost Effective Target Fabrication for Inertial Fusion Energy”

[4]Brian Vermillion, “Microencapsulation Studies for Mass Production of IFE Targets” 


\section{Progress in IFE Technology: June-September 2003 (Cont'd.)}

\section{Target fabrication: Hohlraum materials - LANL/GA}

J. Maxwell, A. Nobile, D. Goodin and R. Petzoldt

- First-ever Growth of Fiber Arrays for High-Z Hohlraum Materials: Developed a new method of fabricating low-density hohlraum materials using diffractive optics to generate multiple reaction zones (See Fig. A), so that many fibers can be grown at once. In this way, a 7x7 array of 49 carbon fibers were grown from ethylene. This demonstrates that it is possible to create lattice-like structures in rapid succession as will be needed for low-density hohlraum materials.

- Calibrated \& used a new apparatus for measuring the temperature at the tip of a fiber, allowing the fiber growth rate to be correlated to the reaction temperature, giving reaction kinetics and thermodynamics. For the first time, the masstransport-limited growth curve was measured for an LCVD reaction, allowing the process to be better controlled (see Fig. B).

- New materials were grown from the vapor phase and the fiber growth rates were measured: Tungsten Carbide Fibers were grown from a mixture of tungsten hexafluoride and ethylene.

- A new method for measuring the temperature distribution across the fiber tip and the fiber diameter was implemented. This uses a chopped infrared image of the fiber tip(s) during growth to give real-time data for controlled growth.

- Invented and implemented a new method of measuring fiber growth rates in realtime, using laser light interference between the focusing objective and fiber tip. In essence this is a FP-interferometer, allowing measurement of the fiber tip position to within a fraction of a wavelength of light. This is perhaps the most important contribution thus far, as a knowledge of the fiber tip location allows us to track the growth for uniform growth.

\section{Publications and Presentations}

[1] J. Maxwell, A. Nobile, D. Goodin, "High-Pressure Laser-induced Synthesis of Carbon Fibers and Microstructures from the Alkenes: Kinetics, Transport, and Thermodynamic Limits" (in Preparation, to be submitted to the Journal Carbon).

[2] J. Maxwell, A. Nobile, D. Goodin, “A Novel Approach to Indirect Drive

Hohlraum Materials, Design, and Mass-Production” (in Preparation, to be submitted to Lasers and Particle Beams).

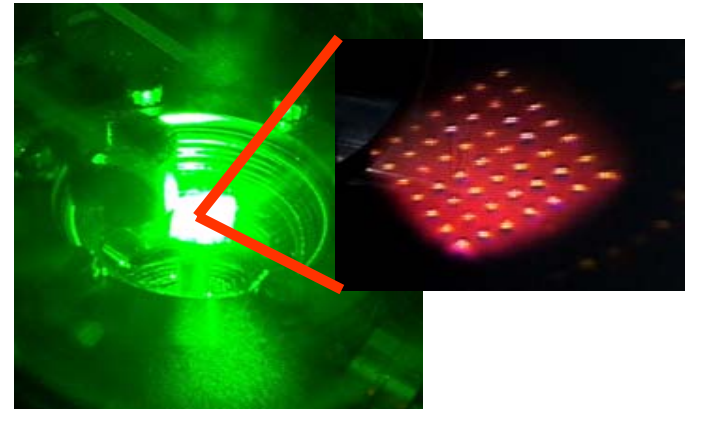

Figure 1. Thermal image of array

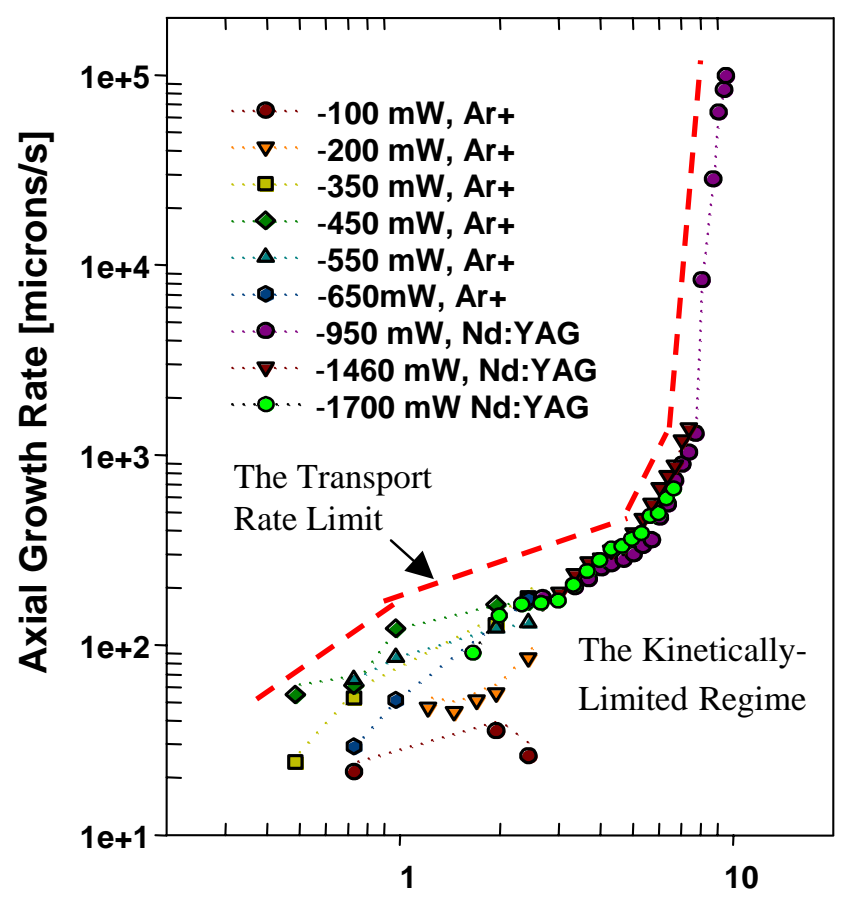

Ethylene Partial Pressure [Bar]

Figure 2. Transport rate limit 


\section{Progress in IFE Technology: June-September 2003 (Cont'd.)}

IFE Chamber Dynamics Modeling and Experiments- University of California, San Diego (http://aries.ucsd.edu/IFE)

M. S. Tillack, F. Najmabadi, A. R. Raffray, S. S. Harilal, C. V. Bindhu, A. C. Gaeris, M. R. Zaghloul, J. O’Shay, K. Sequoia

- We have continued our analysis of the dynamics associated with an expanding laser-produced plasma in a steady state external magnetic field. In the early phase of the plume expansion, the directed beta $\left(\beta_{d}=4 \pi n M^{2} / B^{2}\right)$ is on the order of a few thousand. This indicates that the hot, highly conductive ionized plume has effectively displaced the magnetic field from its volume through the generation of large diamagnetic currents. (See Figure 1.)

- Spectroscopic measurements of the plasma expanding across a transverse magnetic field were used to determine temporal as well as spatial profiles of electron temperature and electron density. In our experiment we observed a 30\% increase in electron temperature near stagnation indicating higher collisionality resulting from confinement of the energetic plume. (See Figure 2.)

- Laser-based diagnostic techniques have been surveyed for in situ time resolved measurment of particle size, velocity, and number density of aerosol particles in IFE relevant ablation plumes. Complete diagnosis of aerosol behavior requires a combination of two or more measurement techniques. Methods such as cavity ring down laser spectroscopy, dark field imaging, and laser induced incandescence have been assessed.

- Work was completed and documented on the propagation of short-pulse lasers in gases beyond the breakdown threshold. Optical emission spectroscopy was used to measure the spatial and temporal evolution of electron density and temperature in air and argon plumes. One article was accepted for publication in Journal of Applied Physics, and another was submitted to Applied Spectroscopy.

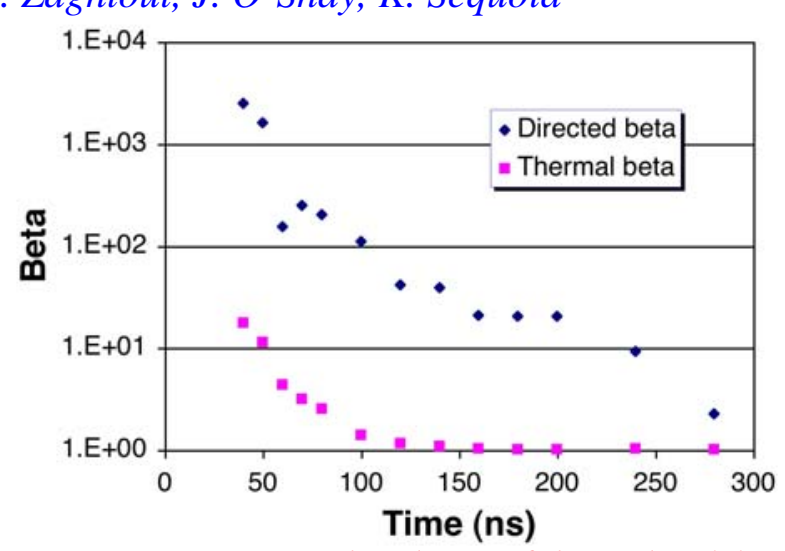

Figure 1. Temporal evolution of thermal and directed beta in the expanding laser-produced plasma

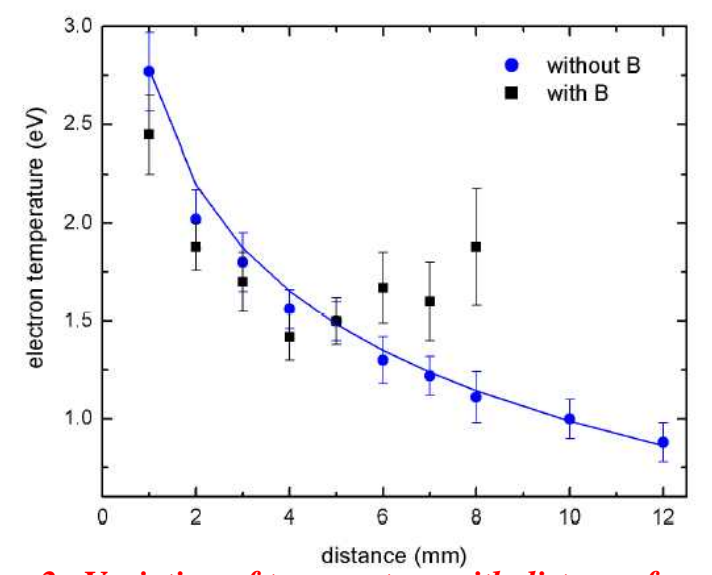

Figure 2. Variation of temperature with distance from the target surface. The solid line is the best-fit curve with $B=0$

\section{Publications and Presentations}

[1] M. S. Tillack, S. S. Harilal, F. Najmabadi and J. O'Shay, "Magnetic confinement of an expanding laser-produced plasma," Inertial Fusion Science and Applications 2003, Monterey CA, Sept. 2003. [2] M. S. Tillack, D. Blair and S. S. Harilal, "The effect of ionization on cluster formation in laser ablation plumes." submitted to Nanotechnology, Aug. 2003. [3] S. S. Harilal, M. S. Tillack, B. O'Shay, C. V. Bindhu, F. Najmabadi, "Confinement and dynamics of laser-produced plasma expanding across a transverse magnetic field," submitted to Phys Rev. E, August 2003. [4] C. V. Bindhu, S. S. Harilal, M. S. Tillack, F. Najmabadi and A. C. Gaeris, "Laser propagation and energy absorption by an argon spark," Journal of Applied Physics 94 (in press, Dec 15, 2003 issue). [5] C. V. Bindhu, S. S. Harilal, M. S. Tillack, F. Najmabadi and A. C. Gaeris, “Energy absorption and propagation in laser created sparks,” submitted to Applied Spectroscopy. [6] A. C. Gaeris, B. Harilal, K. Sequoia and M. S. Tillack, “Aerosol Diagnostics for Liquid-Protected IFE Chambers,” UCSD Technical Report UCSD-ENG-104, October 1, 2003. [7] M. S. Tillack, "Mechanisms of Aerosol Generation in Liquid-Protected IFE Chambers," US-Japan Workshop on Power Plant Studies, Oct. 9-11, 2003. 